\title{
Vortex excitation in a stirred toroidal Bose-Einstein condensate
}

\author{
A. I. Yakimenko, ${ }^{1}$ K. O. Isaieva, ${ }^{1}$ S. I. Vilchinskii, ${ }^{1,2}$ and E. A. Ostrovskaya ${ }^{3}$ \\ ${ }^{1}$ Department of Physics, Taras Shevchenko National University of Kyiv, 64/13 Volodymyrska Str., 01601 Kyiv, Ukraine \\ ${ }^{2}$ Departement de Physique Theorique Center for Astroparticle Physics, Universite de Geneve, \\ Quai E. Ansermet 24, 1211 Geneve 4, Switzerland \\ ${ }^{3}$ Nonlinear Physics Centre, Research School of Physics and Engineering, The Australian National University, Canberra ACT 0200, Australia
}

(Received 20 August 2014; published 9 February 2015)

\begin{abstract}
Motivated by a recent experiment [K. C. Wright et al., Phys. Rev. A 88, 063633 (2013)], we investigate the microscopic mechanism for excitation of vortices and formation of a persistent current in an annular BEC stirred by a narrow blue-detuned optical beam. In the framework of a two-dimensional mean-field model, we study the dissipative dynamics of the condensate with parameters that reflect realistic experimental conditions. Vortex-antivortex pairs appear near the center of the stirrer in the bulk of the condensate for slow motion of the stirring beam. When the barrier angular velocity is above some critical value, an outer edge surface mode develops and breaks into the vortices entering the condensate annulus. We determine the conditions for creation of vortex excitations in the stirred toroidal condensate and compare our results with experimental observations.
\end{abstract}

DOI: 10.1103/PhysRevA.91.023607

PACS number(s): 03.75.Lm, 03.75.Kk, 05.30.Jp

\section{INTRODUCTION}

Nucleation of quantized vortices and their dynamics are closely associated with superflow decay and turbulence in quantum fluids and atomic Bose-Einstein condensates (BECs) [1]. In early experiments, a simple-connected geometry of the trap was used to measure the onset of dissipationless flow in BEC. A laser beam was moved through the condensate, and dissipation was detected as heating of the condensate [2-6]. The problem of vortex excitation by a moving barrier was also extensively investigated theoretically [7-10].

Recent experimental progress in creating atomic gases in a toroidal geometry has opened novel prospects for studies of the fundamental properties of the superfluid state [11]. The ring-shaped BEC has become the topic of a large body of experimental and theoretical research [12-17] including persistent currents [18-20], weak links [21-24], solitary waves [14,25], and decay of a persistent current via phase slips [26]. A persistent flow in a toroidal trap can be created by transferring a quantized angular momentum from optical fields $[18,22]$ or by stirring with a rotating blue-detuned laser beam $[22,27]$. The quantized circulation in a ring corresponds to an $m$-charged vortex line pinned at the center of the ring-shaped condensate, where the vortex energy has a local minimum. Since the vortex core is bounded by the potential barrier, even multicharged $(m>1)$ metastable vortex states can be very robust [26].

Very recently [27], vortices in a toroidal trap were excited using a small (diameter less than the width of the annulus), variable-height potential barrier (a "stirrer") with an angular velocity ranging from 0 up to the speed of sound in the condensate. A wide range of the experimental parameters used in Ref. [27] opens the possibility for theoretical investigation of excitation of a persistent current in a stirred ring-shaped BEC under different regimes. The simple 1D model used in Ref. [27] shows a reasonable agreement with the experimental measurements of the threshold for vortex excitation. However, the microscopic origin of the superflow generation, as well as the complex vortex dynamics, is beyond the scope of the $1 \mathrm{D}$ treatment, which is based on the analysis of the average speed of sound. In this work, we consider a 2D reduction of the full 3D mean-field model justified by the trapping geometry. Performing numerical modeling for various combinations of potential barrier height and angular velocity, we determine the conditions for creation of vortices and compare our results with the experimental findings.

\section{MODEL}

In modeling nonequilibrium behavior, such as nucleation of vortices, dissipative effects are of crucial importance since they provide the mechanism for relaxation to an equilibrium state. It is dissipation that either causes the vortex line to drift to the outer edge of the condensate (where vortices decay) or leads to the pinning of the vortex in the central hole of the ring-shaped condensate. Relaxation of the vortex core position to the local minimum of the energy leads to formation of a metastable persistent current. Dissipative effects appear in a trapped condensate due to interaction with a thermal cloud and can be captured phenomenologically by the dissipative Gross-Pitaevskii equation (GPE) [28-30]. For a system of weakly interacting degenerate atoms close to thermodynamic equilibrium and subject to weak dissipation, the dissipative GPE for the macroscopic wave function can be written in the form

$$
(i-\gamma) \hbar \frac{\partial \tilde{\Psi}(\mathbf{r}, t)}{\partial t}=\left[\hat{H}+\tilde{g}|\tilde{\Psi}(\mathbf{r}, t)|^{2}-\mu\right] \tilde{\Psi}(\mathbf{r}, t),
$$

where $\gamma \ll 1$ is the phenomenological dissipative parameter, $\hat{H}=-\frac{\hbar^{2}}{2 M} \Delta+V(\mathbf{r}), \Delta$ is the Laplace operator, $\tilde{g}=\frac{4 \pi \hbar^{2} a_{s}}{M}$ is the interaction strength, $M$ is the mass of the ${ }^{23} \mathrm{Na}$ atom, and $a_{s}=2.75 \mathrm{~nm}$ is the $s$-wave scattering length. The system described by Eq. (1) conserves neither the energy nor the number of particles. The chemical potential $\mu(t)$ of the equilibrium state in our dynamical simulations was adjusted at each time step so that the number of condensed particles slowly decays with time: $N(t)=N(0) e^{-t / t_{0}}$, where $t_{0}=10 \mathrm{~s}$ corresponds to the $1 / e$ lifetime of the $\mathrm{BEC}$ reported in Ref. [19]. We note that a time-dependent chemical potential was previously used to study quantum hydrodynamics [30]. 
(a)
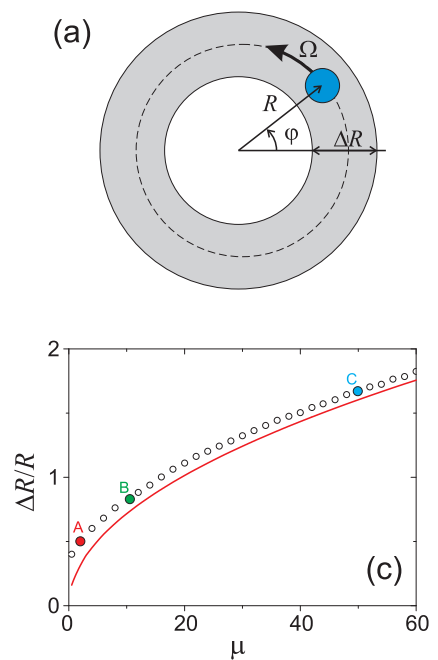
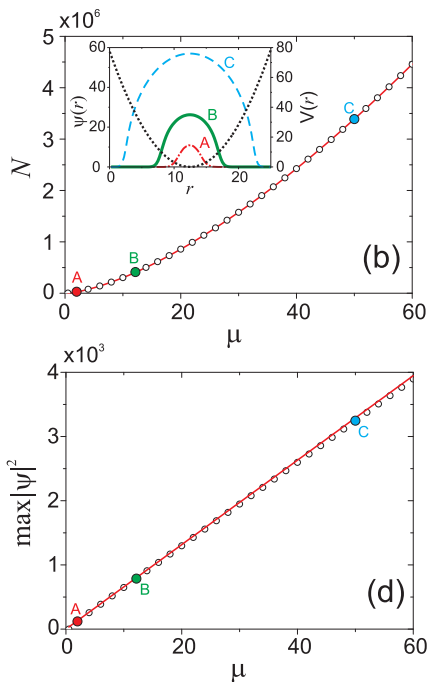

FIG. 1. (Color online) (a) Scheme of the 2D BEC in a ring trap of radius $R$. A blue-detuned optical stirrer [filled (blue) circle] excites vortices when it circles counterclockwise at angular velocity $\Omega$ through the maximum of the condensate density (dashed black line). Stationary ground states. (b) Number of atoms vs chemical potential. Inset: Radial trapping potential $V(r)$ (dotted black line) and examples of numerically found radial profiles $\psi(r)$ for three values of $\mu$ : A, $\mu=2$; B , $\mu=10.62$; and C, $\mu=50$. (c) The solid (red) line shows the relative Thomas-Fermi width $\Delta R / R$ of the ring as a function of the chemical potential. Circles represent numerical results for the relative width $\Delta R / R$ of the condensate with a density above $10^{-3}$ of the peak density. (d) Peak density $\max |\psi|^{2}$ vs $\mu$. The solid (red) line presents the results of the Thomas-Fermi approximation; open circles correspond to numerical stationary solutions.

For numerical simulations corresponding to the experimental condensate holding time, $1 \mathrm{~s}$, changes in the number of atoms are small. However, for some long-term simulations described in this paper the decay of $N$ during the simulation time can be significant and must be taken into account.

The trapping potential,

$$
V(\mathbf{r})=V_{\mathrm{tr}}(r, z)+V_{\mathrm{b}}(x, y, t)
$$

consists of an axially symmetric time-independent toroidal trap,

$$
V_{\mathrm{tr}}(r, z)=\frac{1}{2} M \omega_{z}^{2} z^{2}+\frac{1}{2} M \omega_{r}^{2}(r-R)^{2},
$$

where $r=\sqrt{x^{2}+y^{2}}$, and the repulsive potential of the optical blue-detuned stirring beam,

$$
V_{\mathrm{b}}(x, y, t)=f(t) e^{-\frac{1}{2 d^{2}}\left\{\left[x-x_{0}(t)\right]^{2}+\left[y-y_{0}(t)\right]^{2}\right\}},
$$

where $\left\{x_{0}, y_{0}\right\}=\{R \cos (\Omega t), R \sin (\Omega t)\}$ is the coordinate of the barrier center, moving counterclockwise at a constant angular velocity $\Omega$ through the maximum of the condensate density [see Fig. 1(a)]. The function $f(t)$ describes the temporal changes in the barrier height: $f(t)$ linearly ramps up in the first $0.1 \mathrm{~s}$ of evolution from 0 to $U_{b}$ and remains unchanged for $0.8 \mathrm{~s}$, then in the last $0.1 \mathrm{~s}$ it ramps down to 0 again.

We assume that the system is tightly confined in the $z$ direction by the potential $V_{z}(z)=\frac{1}{2} M \omega_{z}^{2} z^{2}$, so that vortex bending and tilting are suppressed, enabling $2 \mathrm{D}$ vortex

dynamics as pointed out in Ref. [31]. The wave function can be factorized as $\Psi_{j}(\mathbf{r}, t)=\tilde{\Psi}_{j}(x, y, t) \Upsilon(z, t)$, where

$$
\Upsilon(z, t)=\left(l_{z} \sqrt{\pi}\right)^{-1 / 2} e^{-\frac{i}{2} \omega_{z} t-\frac{1}{2} z^{2} / l_{z}^{2}}
$$

is the ground state for the potential $V_{z}(z)$. The norm of the condensate wave function $\tilde{\Psi}(x, y)$ is equivalent to the number of atoms: $N=\int|\tilde{\Psi}|^{2} d x d y$. After integrating out the longitudinal coordinates in the 3D GPE, (1), we obtain a dissipative GPE in 2D,

$$
(i-\gamma) \frac{\partial \psi}{\partial t}=\left[-\frac{1}{2} \Delta_{\perp}+V(x, y, t)+g|\psi|^{2}-\mu\right] \psi,
$$

where $V(x, y, t)=\frac{1}{2}(r-R)^{2}+V_{\mathrm{b}}(x, y, t)$ and $R=12.47$ are the dimensionless potential and the radius of the trap, $g=$ $\sqrt{8 \pi} a_{s} / l_{z}=1.54 \times 10^{-2}$ is the dimensionless $2 \mathrm{D}$ interaction constant, $\psi \rightarrow l_{r} \psi$ is the dimensionless wave function, and $l_{r}=\sqrt{\hbar /\left(M \omega_{r}\right)}$ is the radial oscillator length. Here we use harmonic oscillator units: $t \rightarrow \omega_{r} t,(x, y) \rightarrow\left(x / l_{r}, y / l_{r}\right)$, $V_{\mathrm{b}} \rightarrow V_{\mathrm{b}} /\left(\hbar \omega_{r}\right), \mu \rightarrow \mu /\left(\hbar \omega_{r}\right)$.

\section{GROUND STATE: STATIONARY SOLUTIONS AND SURFACE MODES}

The radially symmetric steady states in toroidal BEC, corresponding to vortices with topological charge $m$, were found by numerically integrating a conservative stationary GPE,

$$
\mu \psi_{m}(r)=-\frac{1}{2} \Delta_{r}^{(m)} \psi_{m}(r)+\frac{1}{2}(r-R)^{2} \psi_{m}(r)+g \psi_{m}^{3}(r),
$$

where

$$
\Delta_{r}^{(m)}=\frac{d^{2}}{d r^{2}}+\frac{1}{r} \frac{d}{d r}-\frac{m^{2}}{r^{2}} .
$$

A ground state with $m=0$ corresponds to the boundary conditions $\psi^{\prime}(0)=0, \psi(\infty)=0$. The numerical results for stationary ground states are given in Fig. 1. Using the Thomas-Fermi (TF) approximation, it is straightforward to obtain the approximate expressions for the number of atoms $N=\frac{4 R}{3 a_{s}}\left(\pi \mu^{3} \omega_{r} / \omega_{z}\right)^{1 / 2}$, width of the BEC annulus $\Delta R=2 \sqrt{2 \mu}$, and peak density $\max |\psi|^{2}=\mu / g$. These simple estimates are found to be in excellent agreement with numerical results (see Fig. 1). As can be seen, the radial profiles gradually expand when the chemical potential increases, and for $\mu>60$ the central hole is filled with the atoms, so that the topology of the condensate becomes single connected.

In the present paper we use the $2 \mathrm{D}$ model that properly describes the dynamical properties of the vortex lines in a toroidal condensate. Obviously, to treat the experiment quantitatively, the correct value for the chemical potential of the $2 \mathrm{D}$ model must be used. We have investigated the process of e persistent current generation for different values of the chemical potential $\mu$ and interaction constant $g$. We note that the threshold for vortex excitation is affected by variation of $\mu$ and $g$, although our main conclusions concerning the generation and evolution of vortex excitations in a stirred ring-shaped condensate do not depend qualitatively on the parameters of the model.

Let us present the results for parameters which are matched to the experimental value of the peak density and geometry of the experimental setup. The corresponding dimensionless value for the chemical potential is $\mu=10.62$. The stationary 
solution for $\mu=10.62$ is shown in the inset in Fig. 1(a) and by the green circles in Figs. 1(b)-1(d). Note that, for the parameters under consideration, the central hole in the ring-shaped condensate is well pronounced, and the width of the annulus $\Delta R=9.22$ is greater than the effective width of the barrier $d=2.1$.

In the toroidal geometry the radial degrees of freedom lead to specific features of the excitations, such as innerand outer-edge surface modes, which cannot be explained in terms of the average speed of sound [32,33]. Recently a Bogoliubov analysis in a $2 \mathrm{D}$ ring system was generalized by including a moving obstacle in Ref. [34]. It appears that the linear eigenmodes corresponding to the critical angular frequency $\Omega_{c}=\min \{\omega(L) / L\}$, obtained by analogy with the Landau criterion (see, e.g., Ref. [35]), are localized at the external surface of the annulus. It is remarkable that the critical frequency $\Omega_{c} / \omega_{r}=\sqrt{2} \mu^{1 / 6} /(R+\Delta R / 2)$ obtained in the framework of the simple surface model (see, e.g., [33]), where the curvature of the external surface is neglected, gives practically the same value, $\Omega_{c}=2 \pi \times 16.1 \mathrm{~Hz}$.

Since stirring by the laser beam is accompanied by condensate distortion and shape deformation, one could expect that a sufficiently rapid barrier (with $\Omega>\Omega_{c}$ ) produces an excited state corresponding to a surface wave with the energy $\hbar \omega(L)$ and angular momentum $\hbar L$ along $z$. Once these surface waves are excited, they break up and nucleate vortices, which come into the condensate bulk from the outer boundary of the annulus. For a slow barrier (with $\Omega<\Omega_{c}$ ) the outer-edge surface mode is not excited and the dominating mechanism for vortex nucleation is expected to be the formation of vortex pairs in the condensate bulk. The direct numerical simulations of the dissipative dynamics of the stirred condensate support these predictions of the linear stability analysis.

\section{DISSIPATIVE DYNAMICS OF A STIRRED TOROIDAL CONDENSATE}

Here we present the results of our numerical simulations of the dissipative GPE, (3), with the split-step Fourier transform method. In line with the experimental procedure, in our calculations, an initially nonrotating condensate is stirred for $1 \mathrm{~s}$ with a repulsive potential moving azimuthally at a fixed angular velocity. In the following, we neglect possible position and temperature dependence of $\gamma$ and set $\gamma=1.5 \times 10^{-3}$. We note that our main results do not depend qualitatively on the chosen value of $\gamma \ll 1$. The dissipative parameter $\gamma$ determines the relaxation time of the vortices: the greater $\gamma$ is, the less time it takes for a vortex to drift from the high-density condensate annulus to the low-density periphery. With $\gamma=1.5 \times 10^{-3}$, we found the bulk of the condensate annulus to be cleansed from the vortices after $3 \mathrm{~s}$, which is consistent with the experimentally measured lifetime of annular vortices [27]. Typical examples of the dissipative dynamics are shown in Fig. 2 for slow and in Fig. 3 for fast rotation of the barrier. More examples of evolution of the density and phase of the toroidal condensate for different angular velocities and heights of the stirring beam are given in the Supplemental Material [36].

We observed two regimes of vortex excitation in a toroidal $\mathrm{BEC}$. For a low angular velocity of the stirrer, vortex-antivortex

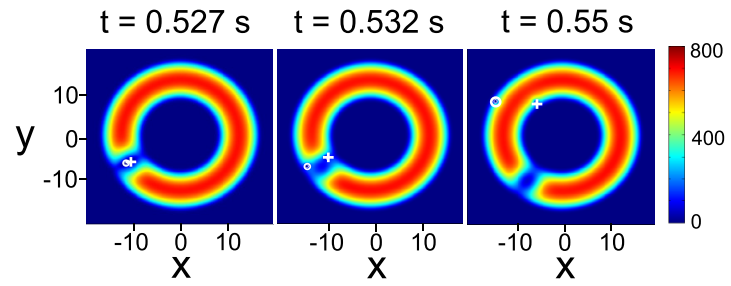

FIG. 2. (Color online) Excitation of a vortex-antivortex pair by the stirring laser beam with $\Omega=2 \pi \times 3 \mathrm{~Hz}$ and $U_{b} / h=1300 \mathrm{~Hz}$. Shown are snapshots of the density distribution for different moments in time. The core of the vortex is indicated by a cross; the antivortex, by a circle.

pairs are nucleated near the center of the barrier (see Fig. 2). Then the pair undergoes a breakdown and the antivortex moves spirally to the external surface of the condensate and, finally, decays into elementary excitations, while the vortex becomes pinned in the central hole of the annulus, adding 1 unit to the topological charge of the persistent current. The progressive drift of the vortices toward the external condensate boundary is the result of dissipation, which leads to vortex energy relaxation.

It is interesting to note the considerable phase gradient traveling in front of the rotating barrier even for a low barrier amplitude and slow rotation, long before the vortex pair appears (see animations in the Supplemental Material [36]). The phase gradient corresponds to the velocity field, which means that a forward superfluid flow appears in the stirred

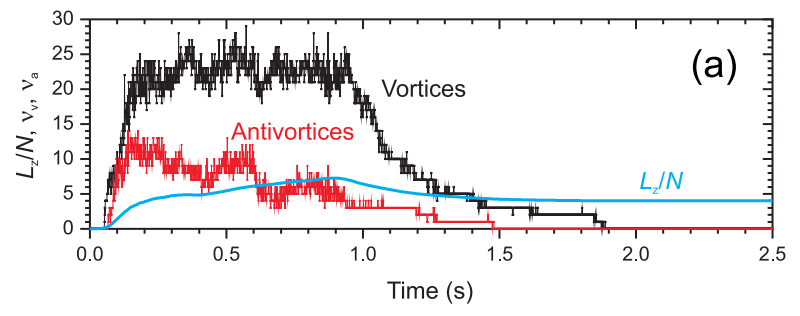

(b)

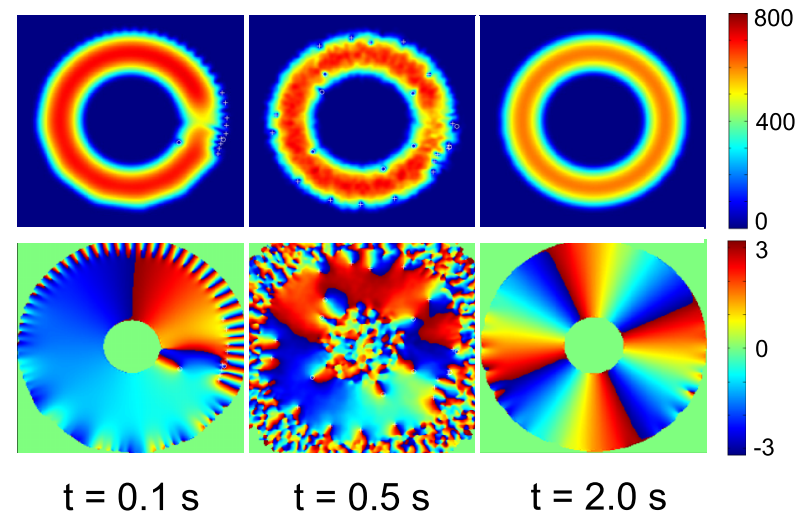

FIG. 3. (Color online) Temporal evolution of the toroidal condensate with a fast stirrer $\left(\Omega=2 \pi \times 20 \mathrm{~Hz}, U_{b} / h=300 \mathrm{~Hz}\right)$. (a) Angular momentum per atom $L_{z} / N$ [solid (blue) curve], number of annular vortices $v_{v}$ (black dots), and number of antivortices $v_{a}$ [gray (red) dots]. (b, c) Snapshots of the density and phase distribution in the $(x, y)$ plane for different moments in time (the size of each image is $40 \times 40$, in units of $l_{r}$ ). 
condensate even well below the threshold rotation rate. This specific feature of the multiply connected superfluid has been conclusively established experimentally in Ref. [37] and can be easily explained as follows: a low-density wake appearing directly behind the stirrer initiates two counter-propagating superflows. The first flow is directed against the rotation of the barrier. This atomic flow tends to fill in the low-density wake behind the barrier. Because the condensate wave function must be single valued, the velocity circulation around any closed path must be a multiple of $2 \pi m$, thus the total velocity circulation vanishes for a state with zero winding number $(m=0)$. The second atomic flow cancels the phase gradient corresponding to the velocity field of atoms moving through the barrier. That is why, in a ring-shaped BEC, a superflow, which is codirectional with the stirrer, appears even for low rotation rates.

It turns out that, if the barrier intensity $U_{b}$ is just above the threshold value, then the angular momentum transfers to the condensate only by nucleation of the vortex-antivortex pair in the bulk of the annulus. Thus, in this regime the moving barrier can be treated as small, in spite of the considerable size of the density dip which it produces in the condensate annulus (see Fig. 2). However, if $U_{b}$ is greater than the threshold value, then a wide weak link (a localized region of rarefied superfluid density) develops in a ring and breaks the potential barrier for external vortices. As a result, a vortex from the outside of the ring can enter though the rotating weak link. This mechanism is similar to the stirring with a wide barrier observed in Ref. [22].

Let us discuss the remarkable analogy between the mechanism of persistent current generation described here and the inverse process of persistent current decay under the influence of a tunable barrier, which was observed experimentally $[18,21]$ and investigated theoretically in the framework of the conservative GPE [23,24] and truncated Wigner approximation [38]. It was revealed in Refs. [23] and [24] that the vortex-antivortex annihilation within the barrier causes the phase slip and decay of the persistent current. In a similar manner the small stirrer at a slow rotation rate and $U_{b}$ close to the threshold value drives the phase slip by vortex dipole nucleation inside the condensate annulus. It is interesting that in the conservative model used in Ref. [23], when the obstacle moves fast with respect to the fluid, instead of vortex annihilation, vortices penetrate the bulk from outside the annulus.

For higher angular velocities of the stirrer (with $\Omega>\Omega_{c}$, where $\Omega_{c}$ is found to be close to the one predicted by Bogoliubov analysis), the dominating source of vortices is the instability of the external surface modes. First, ripples appear at the external surface (see Fig. 3), and then several vortices nucleate simultaneously. Also, as often happens for a higher barrier intensity $U_{b}$, vortex lines come into the bulk of the condensate through the rotating density valley. Further complex dynamics of the vortices is governed not only by the condensate inhomogeneity and dissipation effects, but also by the interplay between condensate flows corresponding to other vortices. Vortex-antivortex annihilation produces sound waves, which also interact with other vortices. Moreover, the sound pulses can also break into vortex-antivortex pairs. As the barrier circles in the annulus, it periodically travels through its own low-density wake, creating new vortices and interacting with existing ones. The number of vortices increases dramatically in our simulations with increasing barrier amplitude $U_{b}$, so that the dynamics of the vortices becomes quite irregular. Note that in the central hole and far from the external surface of the condensate, where the condensate density vanishes, the phase strongly fluctuates and one can see infinitely many vortices and antivortices. To avoid the influence of these "ghost vortices," we discard the contribution to $v_{v}$ and $v_{a}$ of vortices and antivortices which are located beyond the TF surface. Since a host of vortices is located at the periphery of the condensate, the number of annular vortices $v_{v}$ and antivortices $v_{a}$ fluctuates when they cross the boundary of the TF surface [see Fig. 3(a)]. To illustrate the relaxation process and formation of a persistent current in Fig. 3, the time frame of the numerical modeling was substantially extended in comparison to the experiment.

Vortex excitations experience dissipation all the time, which sends them in search of an equilibrium position in accordance with the local minima of the vorticity energy. In an inhomogeneous ring-shaped condensate, the two options are: (i) relaxation to the persistent current, when the vortex line is pinned in the giant central hole, (ii) relaxation to the outer boundary, where vortices decay into elementary excitations. As is well known (see, e.g., [30]), small-scale forcing may generate large-scale flows in effectively 2D turbulent classical and quantum fluids. This feature is in sharp contrast to hydrodynamic turbulence in 3D fluids. Recently [31] it was demonstrated, both experimentally and numerically, for an oblate BEC confined in an annular trapping potential, that inverse energy cascades are inherent for $2 \mathrm{D}$ quantum turbulence. In this context, the formation of large-scale persistent current observed in our simulations is naturally determined by the development of $2 \mathrm{D}$ turbulence. The energy of small-scale forcing first transits to irregular vortex distributions and then relaxes to a large-scale flow in the form of a circulating superflow. For example, as shown in Fig. 3(a), the number of vortices and antivortices rapidly increases when the stirring beam ramps up $(0<t<0.1 \mathrm{~s})$ and decays to 0 when the stirring beam is switched off $(t>1 \mathrm{~s})$, while the angular momentum per atom saturates to an integer number $m=4$ corresponding to the $m$-charged persistent current.

We have performed an extensive series of numerical simulations of a full 1-s operating cycle for different values of barrier intensity $U_{b}$ and angular velocity $\Omega$. To summarize our findings and compare the results of the numerical modeling and the experiment, we present the angular momentum per atom $L_{z} / N$ [see Fig. 4(a)], number of vortices $v_{v}$ [see Fig. 4(b)], and number of antivortices $v_{a}$ [see Fig. 4(c)] inside the condensate annulus at the end of the cycle when the barrier is ramped down to 0 . The relaxation time from a nonequilibrium turbulent state to a metastable state with a persistent current is considerable (comparable with the 3-s lifetime of annular vortices). During $1 \mathrm{~s}$ of the experimental cycle such a transition usually is not completed and the number of annular vortices fluctuates substantially when vortices move towards the condensate edge. To minimize the contribution from these random fluctuations, we have calculated $v_{v}$ [shown in Fig. 4(b)] and $v_{a}$ [shown in 

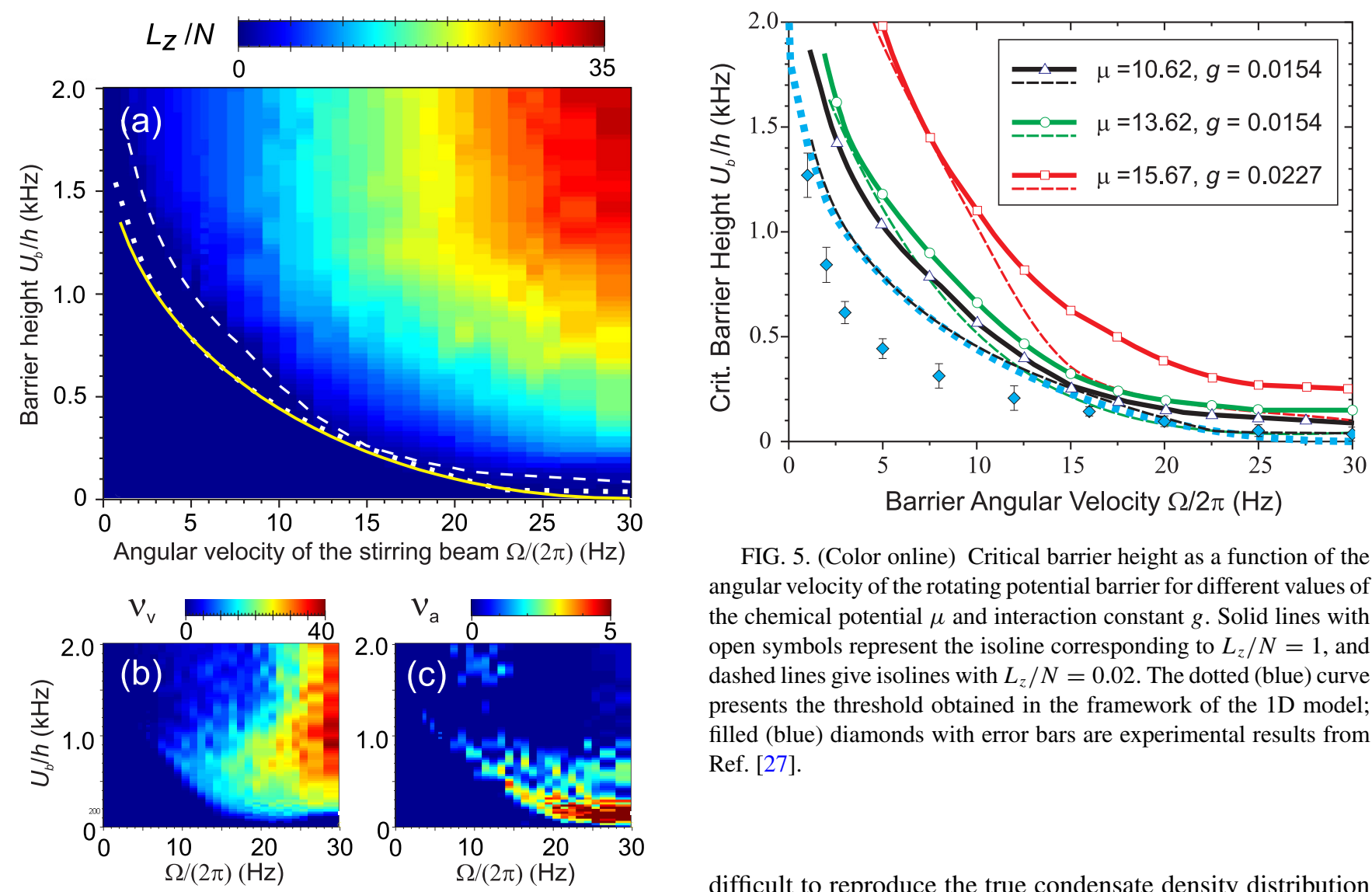

FIG. 4. (Color online) The angular momentum, number of annular vortices, and number of antivortices for the final stage of the stirring process $(t=1 \mathrm{~s})$ are represented by the color code as a function of the angular speed $\Omega$ and the height of the stirring potential $U_{b}$. (a) Angular momentum per atom $L_{z} / N$. White isolines correspond to $L_{z} / N=1$ (dashed curve) and $L_{z} / N=0.02$ (dotted curves). The solid (yellow) curve presents the threshold obtained in the framework of the 1D model used in [27]. (b) Number of annular vortices $v_{v}$. (c) Number of annular antivortices $v_{a}$.

Fig. 4(c)] by averaging the number of annular vortices and antivortices over the period of $10 \mathrm{~ms}$ before the end and $10 \mathrm{~ms}$ after the end of the stirring process. It is interesting to note that the number of detected annular vortices and antivortices decreases for very high values of $U_{b}$. This is because, as mentioned above, a high-amplitude stirring beam creates a large density valley and therefore breaks the potential barrier for external vortices. As a result, a vortex from the outside of the ring readily enters the central hole without crossing the condensate annulus.

The dashed white curve in Fig. 4(a) represents an extrapolated isoline corresponding to the unitary value of the angular momentum per particle $L_{z} / N=1$ (see also Fig. 5). The dotted white line shows the isoline with $L_{z} / N=0.02$, corresponding to the threshold for annular vortex excitation, when a vortex line is placed at the external TF boundary of the annulus. Vortices having an angular momentum above this threshold become visible in time-of-flight images.

As pointed out above, in the framework of the $2 \mathrm{D}$ reduction, which assumes the ground-state distribution, (2), along $z$, it is

FIG. 5. (Color online) Critical barrier height as a function of the angular velocity of the rotating potential barrier for different values of the chemical potential $\mu$ and interaction constant $g$. Solid lines with open symbols represent the isoline corresponding to $L_{z} / N=1$, and dashed lines give isolines with $L_{z} / N=0.02$. The dotted (blue) curve presents the threshold obtained in the framework of the 1D model; filled (blue) diamonds with error bars are experimental results from Ref. [27].

difficult to reproduce the true condensate density distribution both in the $z=0$ plane and along $z$. Since the 2D model should be comparable to the experiment, one needs to adjust the parameters of the model to reproduce the correct condensate peak density or TF radius. We compare the thresholds for three reasonable choices of parameters for the $2 \mathrm{D}$ model in Fig. 5: (i) Black curves give the results for $\mu=10.62$, $g=0.0154$, which reproduce the experimental condensate peak density. It is a natural choice for the narrow stirrer used in the experiment [27], where the vortices are nucleated in the bulk of the condensate. (ii) Green curves represent the results for the $2 \mathrm{D}$ model with parameters $\mu=13.62$ and $g=0.0154$, obtained for the oscillatory wave function (2) along $z$ without fitting $\mu$ to reproduce the condensate peak density. (iii) Red curves demonstrate the results of the $2 \mathrm{D}$ model, which reproduces the correct TF radius. This can be achieved by adjusting the interaction constant and assuming that the chemical potential in the $2 \mathrm{D}$ model is equal to its $3 \mathrm{D}$ counterpart: $\mu=\mu_{3 \mathrm{D}}=15.67$ and $g=0.0227$.

Surprisingly, all the predictions obtained for the 2D model give overestimated thresholds in comparison with the experimental measurements and are never better than those obtained for the 1D model used in [27]. It turns out that the 2D dissipative GPE describes the experiment only qualitatively in the slow-stirring regime. The agreement with experiment is better in the fast-stirring regime, however, even matching the $\mathrm{TF}$ radii does not help us to reproduce the threshold for persistent current excitation with the accuracy rate reported in the experiment [27].

The problem of persistent current formation in a stirred toroidal condensate deserves further theoretical and experimental investigation. In particular, the influence of the 
inevitable experimental shot-to-shot deviation in the number of atoms and impurities in the condensate density on phase slips should be analyzed in more detail. Furthermore, the question is open whether the regime where vortex-antivortex pairs are generated in the bulk is ever reached in the experiment since no choice of parameters produces agreement with the experiment for a slow rotation rate. In our numerical simulations, we have observed that even a relatively small deviation $\delta R$ of the stirring beam center trajectory $(\delta R \approx$ $2 \mu \mathrm{m}$, which is of the order of the precision of stable beam axis positioning reported in Ref. [3]) from the ideal circle of radius $R=22.6 \mu \mathrm{m}$ can significantly decrease the threshold barrier height $U_{b}$ for a slowly rotating stirrer. For example, in the Supplemental Material [36] we present two simulations with the trajectories of the stirring beam described by $r=R(1+\varepsilon \cos \varphi)$. For the undistorted circle $(\varepsilon=0)$ no phase slip occurs, while for the simulation with the same parameters but with $\varepsilon=0.1$ a two-charged persistent current is generated.

It is, furthermore, instructive to generalize our 2D theoretical model to a realistic 3D geometry. In the real 3D geometry at the rotating barrier center the TF height of the condensate decreases with $U_{b}$ as $\sqrt{1-U_{b} / \mu}$. Since the energy of a vortex is proportional to the length of the vortex line, accounting for this effect can significantly reduce the threshold for vortex nucleation. The influence of the stochastic thermal fluctuations on vortex nucleation close to the threshold rotation rate merits a separate study. The role of thermal fluctuations can be especially significant with slow stirring, since the critical barrier height is larger (thus the local density is more depleted), allowing thermal atoms to fill a low-density region. Also, it is of interest to investigate, both experimentally and theoretically, the relaxation process to a metastable persistent current when the condensate is cleansed from annular vortices depending on the position of the stirring beam.

\section{CONCLUSIONS}

We have investigated a toroidal condensate stirred by a small, tunable, penetrable barrier moving azimuthally at a fixed angular velocity. Our theoretical analysis reveals the microscopic mechanism for large-scale persistent current generation and demonstrates a small-scale complex vortex dynamics. In particular, we describe and delineate two regimes of vortex nucleation for a slow- and a fast-moving stirrer. For a slow motion of the narrow stirring beam vortex-antivortex pairs appear near the center of the stirrer in the bulk of the condensate. The antivortex moves spirally to the external surface of the condensate and, finally, decays into elementary excitations, while the vortex becomes pinned in the central hole of the annulus, adding one unit to the topological charge of the persistent current. When the barrier angular velocity is above some critical angular velocity, in accordance with the results of Bogoliubov analysis and the predictions of the surface model, an outer-edge surface mode develops and breaks into the vortices entering the condensate annulus.

Performing numerical modeling for various combinations of potential barrier height and angular velocity, we determine conditions for the creation of vortices which appear to be in qualitative agreement with the experimental results [27]. We believe that our predictions can be further tested in the ongoing experiments with atomtronic circuits.

\section{ACKNOWLEDGMENTS}

The authors thank M. Weyrauch and Yu. M. Bidasyuk for useful discussions. E.A.O. acknowledges support from the Australian Research Council (ARC). S.V. is grateful to the Swiss National Science Foundation (individual Grant No. IZKOZ2_154984) and to Prof. Ruth Durrer for her support and kind hospitality.
[1] A. L. Fetter and A. A. Svidzinsky, J. Phys. Condens. Matter 13, R135 (2001).

[2] C. Raman, M. Köhl, R. Onofrio, D. S. Durfee, C. E. Kuklewicz, Z. Hadzibabic, and W. Ketterle, Phys. Rev. Lett. 83, 2502 (1999).

[3] K. W. Madison, F. Chevy, W. Wohlleben, and J. Dalibard, Phys. Rev. Lett. 84, 806 (2000).

[4] R. Onofrio, C. Raman, J. M. Vogels, J. R. Abo-Shaeer, A. P. Chikkatur, and W. Ketterle, Phys. Rev. Lett. 85, 2228 (2000).

[5] C. Raman, J. R. Abo-Shaeer, J. M. Vogels, K. Xu, and W. Ketterle, Phys. Rev. Lett. 87, 210402 (2001).

[6] T. W. Neely, E. C. Samson, A. S. Bradley, M. J. Davis, and B. P. Anderson, Phys. Rev. Lett. 104, 160401 (2010).

[7] B. Jackson, J. F. McCann, and C. S. Adams, Phys. Rev. Lett. 80, 3903 (1998).

[8] B. Jackson, J. F. McCann, and C. S. Adams, Phys. Rev. A 61, 051603 (2000).

[9] J. S. Stießberger and W. Zwerger, Phys. Rev. A 62, 061601 (2000).

[10] T. Aioi, T. Kadokura, T. Kishimoto, and H. Saito, Phys. Rev. X 1, 021003 (2011).
[11] N. Murray, M. Krygier, M. Edwards, K. C. Wright, G. K. Campbell, and C. W. Clark, Phys. Rev. A 88, 053615 (2013).

[12] K. Kasamatsu, M. Tsubota, and M. Ueda, Phys. Rev. A 66, 053606 (2002).

[13] M. Benakli, S. Raghavan, A. Smerzi, S. Fantoni, and S. R. Shenoy, Europhys. Lett. 46, 275 (1999).

[14] J. Brand and W. P. Reinhardt, J. Phys. B 34, L113 (2001).

[15] A. Das, J. Sabbatini, and W. H. Zurek, Sci. Rep. 2, 352 (2012).

[16] J.-P. Martikainen, K.-A. Suominen, L. Santos, T. Schulte, and A. Sanpera, Phys. Rev. A 64, 063602 (2001).

[17] M. Modugno, C. Tozzo, and F. Dalfovo, Phys. Rev. A 74, 061601 (2006).

[18] C. Ryu, M. F. Andersen, P. Cladé, V. Natarajan, K. Helmerson, and W. D. Phillips, Phys. Rev. Lett. 99, 260401 (2007).

[19] S. Beattie, S. Moulder, R. J. Fletcher, and Z. Hadzibabic, Phys. Rev. Lett. 110, 025301 (2013).

[20] A. I. Yakimenko, K. O. Isaieva, S. I. Vilchinskii, and M. Weyrauch, Phys. Rev. A 88, 051602 (2013).

[21] A. Ramanathan, K. C. Wright, S. R. Muniz, M. Zelan, W. T. Hill, III, C. J. Lobb, K. Helmerson, W. D. Phillips, and G. K. Campbell, Phys. Rev. Lett. 106, 130401 (2011). 
[22] K. C. Wright, R. B. Blakestad, C. J. Lobb, W. D. Phillips, and G. K. Campbell, Phys. Rev. Lett. 110, 025302 (2013).

[23] F. Piazza, L. A. Collins, and A. Smerzi, Phys. Rev. A 80, 021601 (2009).

[24] F. Piazza, L. A. Collins, and A. Smerzi, J. Phys. B 46, 095302 (2013).

[25] P. Mason and N. G. Berloff, Phys. Rev. A 79, 043620 (2009).

[26] S. Moulder, S. Beattie, R. P. Smith, N. Tammuz, and Z. Hadzibabic, Phys. Rev. A 86, 013629 (2012).

[27] K. C. Wright, R. B. Blakestad, C. J. Lobb, W. D. Phillips, and G. K. Campbell, Phys. Rev. A 88, 063633 (2013).

[28] L. Pitaevskii, Zh. Eksp. Teor. Fiz. 35, 408 (1958) [Sov. Phys. JETP 35, 282 (1959)].

[29] S. Choi, S. A. Morgan, and K. Burnett, Phys. Rev. A 57, 4057 (1998).

[30] M. Tsubota, M. Kobayashi, and H. Takeuchi, Phys. Rep. 522, 191 (2013).
[31] T. W. Neely, A. S. Bradley, E. C. Samson, S. J. Rooney, E. M. Wright, K. J. H. Law, R. Carretero-González, P. G. Kevrekidis, M. J. Davis, and B. P. Anderson, Phys. Rev. Lett. 111, 235301 (2013).

[32] S. J. Woo and Y.-W. Son, Phys. Rev. A 86, 011604 (2012).

[33] R. Dubessy, T. Liennard, P. Pedri, and H. Perrin, Phys. Rev. A 86, 011602 (2012).

[34] M. Kunimi and Y. Kato, arXiv:1407.7915.

[35] F. Dalfovo and S. Stringari, Phys. Rev. A 63, 011601(R) (2000).

[36] See Supplemental Material at http://link.aps.org/supplemental/ 10.1103/PhysRevA.91.023607 for examples of animations of dynamics of the stirred toroidal condensate.

[37] S. Eckel, F. Jendrzejewski, A. Kumar, C. J. Lobb, and G. K. Campbell, Phys. Rev. X 4, 031052 (2014).

[38] A. C. Mathey, C. W. Clark, and L. Mathey, Phys. Rev. A 90, 023604 (2014). 